\title{
The Isotopic Foldy-Wouthuysen Representation and Chiral Symmetry. The Standard Model in the Isotopic Foldy-Wouthuysen Representation without Higgs Bosons in the Fermion Sector.
}

\section{V.P.Neznamov ${ }^{1}$}

Russian Federal Nuclear Center - The All-Russian Research Institut of Experimental Physics Sarov, N.Novgorod Region, 37 Mira Pr., 607188, Russia

E-mail: neznamov@vniief.ru

The report introduces the isotopic Foldy-Wouthuysen representation. This representation was used to derive chiral invariant equations for massive interacting fermion fields.

In the isotopic Foldy-Wouthuysen representation, the ground state of fermions(vacuum) turns out to be degenerate, and therefore there is the possibility of spantaneously breaking parity (P-symmetry).

The Standard Model with massive fermions is formulated in the isotopic FoldyWouthuysen representation. $\mathrm{SU}(2) \mathrm{xU}(1)$ - invariance of the theory in this representation is independent of whether fermions possess mass or not, and, consequently, it is not necessary to introduce interactions between Higgs bosons and fermions.

The XXth International Workshop High Energy Physics and Quantum Field Theory

Sochi, Russia

September 24-October 1, 2011

Speaker 


\section{Introduction}

As we know in the original Standard Model is considered massless fermions to ensure the $S U(2)$ invariance. Masses are imparted to fermions after the introduction of the mechanism of spontaneous breaking of symmetry, the resulting occurrence of Higgs bosons and postulation of their gauge-invariant interactions with fermions with Yukawa coupling.

There is a question of possibility of formulation of $\mathrm{SU}(2) \mathrm{xU}(1)$ - invariant Standard model without use of Higgs bosons in fermion sector and with conservation of all other its theoretical forecast and consequences.

In this formulation, fermion masses are introduced from outside, as it had usually been done in quantum fields theory modifications before the occurrence of the Standard model.

In this report, we answered the questions stated above in the affirmative by introducing a special isotopic Foldy-Wouthuysen representation.

\section{Chirally symmetric equations for massive fermions in the Dirac representation}

Let us consider the Hamiltonian density of a Dirac particle with mass $m$, which interacts with an arbitrary vector boson field $B^{\mu}(x)$

$$
\begin{aligned}
& \mathcal{H}_{D}=\psi^{\dagger}\left(\boldsymbol{\alpha} \mathbf{p}+\beta m+q \alpha_{\mu} B^{\mu}\right) \psi= \\
& =\psi^{\dagger}\left(P_{L}+P_{R}\right)\left(\boldsymbol{\alpha} \mathbf{p}+\beta m+q \alpha_{\mu} B^{\mu}\right)\left(P_{L}+P_{R}\right) \psi= \\
& =\psi_{L}^{\dagger}\left(\boldsymbol{\alpha} \mathbf{p}+q \alpha_{\mu} B^{\mu}\right) \psi_{L}+\psi_{R}^{\dagger}\left(\boldsymbol{\alpha} \mathbf{p}+q \alpha_{\mu} B^{\mu}\right) \psi_{R}+\psi_{L}^{\dagger} \beta m \psi_{R}+\psi_{R}^{\dagger} \beta m \psi_{L}
\end{aligned}
$$

In equalities (1) $q$ - is the coupling constant; $\alpha^{i}, \beta$ - матрицы Дирака, $\alpha^{\mu}=\left\{\begin{array}{l}1 \quad \mu=0 \\ \alpha^{i} \quad \mu=i=1,2,3\end{array} ; P_{L}=\frac{1-\gamma_{5}}{2}, P_{R}=\frac{1+\gamma_{5}}{2}-\right.$ are the left and right projection operators; $\psi_{L}=P_{L} \psi, \quad \psi_{R}=P_{R} \psi-$ are the left and right components of the Dirac field operator $\psi$.

The Hamiltonian density $\mathcal{H}_{D}$ allows obtaining the motion equations for $\psi_{L}$ and $\psi_{R}$

$$
\begin{aligned}
& p_{0} \psi_{L}=\left(\boldsymbol{\alpha p}+q \alpha_{\mu} B^{\mu}\right) \psi_{L}+\beta m \psi_{R} \\
& p_{0} \psi_{R}=\left(\boldsymbol{\alpha p}+q \alpha_{\mu} B^{\mu}\right) \psi_{R}+\beta m \psi_{L}
\end{aligned}
$$


One can see that both Hamiltonian density $\mathcal{H}_{D}$ (1) and Eqs. (2) have such a form that the presence of mass in fermions results in mixing the right and left components of the field operator $\psi$, and chiral symmetry and $\mathrm{SU}(2) \mathrm{xU}(1)$ - symmetry is therefore preserved only for massless fermions.

Now, let us question ourselves, whether it is possible to write chirally symmetric equations and their Hamiltonians for massive fermions.

It follows from Eqs. (2) that

$$
\begin{aligned}
& \psi_{L}=\left(p_{0}-\boldsymbol{\alpha} \mathbf{p}-q \alpha_{\mu} B^{\mu}\right)^{-1} \beta m \psi_{R} \\
& \psi_{R}=\left(p_{0}-\boldsymbol{\alpha} \mathbf{p}-q \alpha_{\mu} B^{\mu}\right)^{-1} \beta m \psi_{L}
\end{aligned}
$$

By substituting (3) to the right-hand part of Eqs. (2) proportional to $\beta m$, we obtain integro-differential equations for $\psi_{R}$ and $\psi_{L}$

$$
\left[\left(p_{0}-\boldsymbol{\alpha} \mathbf{p}-q \alpha_{\mu} B^{\mu}\right)-\left(p_{0}+\boldsymbol{\alpha} \mathbf{p}-q \boldsymbol{\alpha}_{\mu} B^{\mu}\right)^{-1} m^{2}\right] \psi_{L, R}=0 ; \quad \boldsymbol{\alpha}_{\mu}=\left\{\begin{array}{l}
1 \\
-\alpha^{i}
\end{array}\right.
$$

In expression (4), designation $\psi_{L, R}$ shows that equations for $\psi_{L}$ and $\psi_{R}$ have the same form.

If we multiply Eqs. (4) on the left by the term $p_{0}+\boldsymbol{\alpha p}-q \boldsymbol{\alpha}_{\mu} B^{\mu}$, we obtain secondorder equations with respect to $p^{\mu}$

$$
\left[\left(p_{0}+\boldsymbol{\alpha} \mathbf{p}-q \boldsymbol{\alpha}_{\mu} B^{\mu}\right)\left(p_{0}-\boldsymbol{\alpha p}-q \alpha_{\mu} B^{\mu}\right)-m^{2}\right] \psi_{L, R}=0
$$

For the case of quantum electrodynamics, $\left(q=e, B^{\mu}=A^{\mu}\right)$, Eqs. (5) take the form of

$$
\left[\left(p_{0}-e A_{0}\right)^{2}-(\mathbf{p}-e \mathbf{A})^{2}-m^{2}+e \mathbf{\Sigma} \mathbf{H}+i e \boldsymbol{\alpha} \mathbf{E}\right] \psi_{L, R}=0
$$

In Eqs. (6) $\mathbf{H}=\operatorname{rot} \mathbf{A}-$ is magnetic field, and $\mathbf{E}=-\frac{\partial \mathbf{A}}{\partial t}-\nabla A_{0}$ is electrical field.

Eqs. (6) coincide with the second-order equation obtained by Dirac in the 1920s for the bispinor $\psi$. The Eqs. (6) for all right and left components $\psi$ were obtained for the first time by Feynman and Gell-Mann in 1958 (Phys. Rev., 109, 193). 


\section{Isotopic Foldy-Wouthuysen representation for massive fermions}

The linear form of the chirally symmetric equations of fermion fields with respect to $p_{0}$ can be derived using the Foldy-Wouthuysen transformation in a specially introduced isotopic space.

Let us introduce an eight-component field operators, $\Phi_{1}=\left(\begin{array}{l}\psi_{R} \\ \psi_{L}\end{array}\right), \quad \Phi_{2}=\left(\begin{array}{l}\psi_{L} \\ \psi_{R}\end{array}\right)$, and isotopic matrices, $\tau_{3}=\left(\begin{array}{cc}E_{4 \times 4} & 0 \\ 0 & -E_{4 \times 4}\end{array}\right), \tau_{1}=\left(\begin{array}{cc}0 & E_{4 \times 4} \\ E_{4 \times 4} & 0\end{array}\right)$ affecting the four upper and four lower components of the operators $\Phi_{1}, \Phi_{2}$.

The Dirac equations for $\Phi_{1}, \Phi_{2}$ can be written as

$$
\left.\begin{array}{l}
p_{0} \psi_{L}=\left(\boldsymbol{\alpha p}+q \alpha_{\mu} B^{\mu}\right) \psi_{L}+\beta m \psi_{R} \\
p_{0} \psi_{R}=\left(\boldsymbol{\alpha p}+q \alpha_{\mu} B^{\mu}\right) \psi_{R}+\beta m \psi_{L}
\end{array}\right\} \rightarrow p_{0} \Phi_{1,2}=\left(\boldsymbol{\alpha p}+\tau_{1} \beta m+q \alpha_{\mu} B^{\mu}\right) \Phi_{1,2}
$$

Let us find the Foldy-Wouthuysen transformation in the isotopic space for Dirac equations (7) without interaction.

$$
\left(U_{0}\right)_{I F W}=U_{E r}=\sqrt{\frac{E+\tau_{3} \mathbf{\alpha p}}{2 E}}\left(1+\frac{1}{E+\tau_{3} \mathbf{\alpha p}} \tau_{3} \tau_{1} \beta m\right)
$$

Transformation (8) is unitary $\left(\left(U_{0}\right)_{I F W}\left(U_{0}\right)_{I F W}^{\dagger}=1\right)$, and

$$
\left(H_{0}\right)_{I F W}=\left(U_{0}\right)_{I F W}\left(\boldsymbol{\alpha p}+\tau_{1} \beta m\right)\left(U_{0}\right)_{I F W}^{\dagger}=\tau_{3} E
$$

Thus, Eqs. (7) in the isotopic Foldy-Wouthuysen representation have the form

$$
p_{0}\left(\Phi_{1,2}\right)_{I F W}=\tau_{3} E\left(\Phi_{1,2}\right)_{I F W}
$$

By applying the transformation matrix $\left(U_{0}\right)_{I F W}(8)$ to $\Phi_{1}, \Phi_{2}$ we obtain 


$$
\begin{aligned}
& \Phi_{1 I F W}^{(+)}(\mathbf{x}, t)=\left(U_{0}\right)_{I F W} \Phi_{1}^{(+)}(\mathbf{x}, t)=e^{-i E t}\left(\begin{array}{c}
\sqrt{\frac{2 E}{E+\Sigma \mathbf{p}}} \psi_{R}^{(+)}(\mathbf{x}) \\
0
\end{array}\right)=e^{-i E t} \frac{1}{\sqrt{2}}\left(\begin{array}{c}
\varphi^{(+)}(\mathbf{x}) \\
\varphi^{(+)}(\mathbf{x}) \\
0 \\
0
\end{array}\right) \\
& \Phi_{1 I F W}^{(-)}(\mathbf{x}, t)=\left(U_{0}\right)_{I F W} \Phi_{1}^{(-)}(\mathbf{x}, t)=e^{i E t}\left(\sqrt{\frac{2 E}{E+\Sigma \mathbf{p}}} \psi_{L}^{(-)}(\mathbf{x})\right)=e^{i E t} \frac{1}{\sqrt{2}}\left(\begin{array}{c}
0 \\
0 \\
-\chi^{(-)}(\mathbf{x}) \\
\chi^{(-)}(\mathbf{x})
\end{array}\right) \\
& \Phi_{2 I F W}^{(+)}(\mathbf{x}, t)=\left(U_{0}\right)_{I F W} \Phi_{2}^{(+)}(\mathbf{x}, t)=e^{-i E t}\left(\sqrt{\frac{2 E}{E-\mathbf{\Sigma} \mathbf{p}}} \psi_{L}^{(+)}(\mathbf{x})\right)=e^{-i E t} \frac{1}{\sqrt{2}}\left(\begin{array}{c}
\varphi^{(+)}(\mathbf{x}) \\
-\varphi^{(+)}(\mathbf{x}) \\
0 \\
0
\end{array}\right) \\
& \Phi_{2 I F W}^{(-)}(\mathbf{x}, t)=\left(U_{0}\right)_{I F W} \Phi_{2}^{(-)}(\mathbf{x}, t)=e^{i E t}\left(\begin{array}{c}
0 \\
\sqrt{\frac{2 E}{E-\Sigma \mathbf{p}}} \psi_{R}^{(-)}(\mathbf{x})
\end{array}\right)=e^{i E t} \frac{1}{\sqrt{2}}\left(\begin{array}{c}
0 \\
0 \\
\chi^{(-)}(\mathbf{x}) \\
\chi^{(-)}(\mathbf{x})
\end{array}\right) .
\end{aligned}
$$

In the presence of boson fields $B^{\mu}(x)$ interacting with fermion fields $\Phi_{1}(x), \Phi_{2}(x)$, the isotopic Foldy-Wouthuysen transformation and the Hamiltonians in the IFW representation can be obtained as a series in powers of the coupling constant (V.P.Neznamov. Voprosi Atomnoy Nauki I Techniki (1988); Physics of Elementary Particles and Atomic Nuclei (2006)).

As a result we obtain

$$
\begin{gathered}
\left(\Phi_{1,2}\right)_{I F W}=U_{I F W} \Phi_{1,2} ; \\
U_{I F W}=\left(1+\delta_{1}+\delta_{2}+\delta_{3}+\ldots\right)\left(U_{0}\right)_{I F W} ; \\
p_{0}\left(\Phi_{1,2}\right)_{I F W}=\left(\tau_{3} E+K_{1}+K_{2}+K_{3}+\ldots\right)\left(\Phi_{1,2}\right)_{I F W} ; \\
\mathcal{H}_{I F W}^{I, I I}=\left(\Phi_{1,2}\right)_{I F W}^{\dagger}\left(\tau_{3} E+K_{1}+K_{2}+K_{3}+\ldots\right)\left(\Phi_{1,2}\right)_{I F W} .
\end{gathered}
$$

Let us introduce a sixteen-component spinor and generalization of the isotopic matrices $\tau_{1}, \tau_{3}$ for return to primary state space of Dirac equations after transition to IFW representation 


$$
\begin{aligned}
& \Phi=\left(\begin{array}{l}
\Phi_{1} \\
\Phi_{2}
\end{array}\right)=\left(\begin{array}{l}
\psi_{R} \\
\psi_{L} \\
\psi_{L} \\
\psi_{R}
\end{array}\right) ; E_{16 \times 16} \cdot \tau_{1}=\sum_{1}^{I} ; E_{16 \times 16} \cdot \tau_{3}=\sum_{3}^{I} ; \rho_{1}^{I} \cdot \tau_{1}=\alpha_{1}^{I} ; \\
& \rho_{1}^{I}=\left(\begin{array}{cc}
0 & E_{8 \times 8} \\
E_{8 \times 8} & 0
\end{array}\right) ; \rho_{3}^{I}=\left(\begin{array}{cc}
E_{8 \times 8} & 0 \\
0 & -E_{8 \times 8}
\end{array}\right) ; \rho_{2}^{I}=i \rho_{3}^{I} \rho_{1}^{I} .
\end{aligned}
$$

The Dirac equation for $\Phi(x)$ and $\Phi_{I F W}(x)$ can be written as

$$
\begin{aligned}
& p_{0} \Phi(x)=\left(\boldsymbol{\alpha p}+\sum_{1}^{I} \beta m+\frac{1}{2}\left(E_{16 \times 16}+\alpha_{1}^{I}\right) q \alpha_{\mu} \beta^{\mu}\right) \Phi(x) \\
& p_{0}(\Phi)_{I F W}=\left(\tau_{3} E+K_{1}+K_{2}+K_{3}+\ldots\right)(\Phi)_{I F W} .
\end{aligned}
$$

The analysis shows that the equations of fermion fields and their Hamiltonians in the isotopic Foldy-Wouthuysen representation for the case of the considered interaction are written in the chirally symmetric form irrespective of whether fermions possess mass or are massless.

\section{The Standard Model in the isotopic Foldy-Wouthuysen respresentation} (V.P.Neznamov, Physics of Elementary Particles and Atomic Nuclei, vol.1 (2012), arxiv:1107.0691, arxiv:1107.0693)

1) Primary we write the quark-lepton Hamiltonian of the Standard model with nonzero fermion masses and with glance of interaction with photons, weak gauge bosons and gluons in accordance with covariant derivative

$$
D_{\mu}=\partial_{\mu}-i g_{1} \frac{Y}{2} \beta_{\mu}-i g_{2} \frac{\tau_{w}^{i}}{2} W_{\mu}^{i}-i g_{3} \frac{\lambda^{\alpha}}{2} G_{\mu}^{\alpha}
$$

We write the Hamiltonian separately for $\mathrm{SU}(2) \mathrm{xU}(1)$ doublets and singlets taking into account that the third summand in Eq. (16), namely $\left(-i g_{2} \frac{1}{2}\left(\tau_{w}^{l} W_{\mu}^{1}+\tau_{w}^{2} W_{\mu}^{2}\right)\right)$, is absent in singlet expressions. As far as fermion masses are not equal to zero, so in such record the theory is not invariant relative to $\mathrm{SU}(2) \mathrm{xU}(1)$ - symmetry.

2) Then we introduce a sixteen-component spinors $\Phi_{D}, \Phi_{S}$ for all doublets and singlets.

3) We make a transition to the Foldy-Wouthuysen representation. Consequently we obtain the Hamiltonian with massive fermions written in chiral-symmetric view and having $\mathrm{SU}(2) \mathrm{xU}(1)$ - symmetry. 
4) For return to interactions of the Standard model the right components $\left(\Phi_{D I F W}\right)_{R}$ for all fermions should necessarily be zero in the summands of the Hamiltonian with doublets $\left(\Phi_{D}\right)_{I F W}$. Contrary, in the summands with singlets should be $\left(\Phi_{S I F W}\right)_{L}=0$ for all fermions. In addition, for all neutrino $\left(v_{e}, v_{\mu}, v_{\tau}\right)$,

$$
\left(\Phi_{D I F W}^{v_{i}}\right)_{R}=0 ; \quad m_{v_{i}}=0
$$

Consequently, the $\mathrm{SU}(2) \mathrm{xU}(1)$ - invariant Standard model with massive fermions without postulation of Yukawa interaction of Higgs bosons with leptons and quark can be formulated in the isotopic Foldy-Wouthuysen representation. Because of the unitarity of the IFW - transformation all the rest of forecasts and consequences of the Standard model were preserved. 